OPEN ACCESS

Edited by:

Shengli $L i$,

Shanghai Jiao Tong University, China

Reviewed by:

Sijia Yan,

Shanghai Jiao Tong University, China

Hong Wang,

China Pharmaceutical University,

China

*Correspondence:

Li Yin

liyin1966zzu@hotmail.com

Specialty section: This article was submitted to

Computational Genomics,

a section of the journa

Frontiers in Genetics

Received: 06 June 2021 Accepted: 02 August 2021 Published: 27 August 2021

Citation:

Li S, Wang $H$, Zhang Y, Qiao R,

Xia $P$, Kong $Z$, Zhao $H$ and Yin $L$ (2021) COL3A1 and MMP9 Serve as

Potential Diagnostic Biomarkers of Osteoarthritis and Are Associated

With Immune Cell Infiltration.

Front. Genet. 12:721258

doi: 10.3389/fgene.2021.721258

\section{COL3A1 and MMP9 Serve as Potential Diagnostic Biomarkers of Osteoarthritis and Are Associated With Immune Cell Infiltration}

\author{
Shushan Li, Haitao Wang, Yi Zhang, Renqiu Qiao, Peige Xia, Zhiheng Kong, \\ Hongbo Zhao and Li Yin*
}

Department of Orthopedic Surgery, The First Affiliated Hospital of Zhengzhou University, Zhengzhou, China

Background: Osteoarthritis $(\mathrm{OA})$ is one of the most common age-related degenerative diseases. In recent years, some studies have shown that pathological changes in the synovial membrane occur earlier than those in the cartilage in OA. However, the molecular mechanism of synovitis in the pathological process of OA has not been elucidated. This study aimed to identify novel biomarkers associated with OA and to emphasize the role of immune cells in the pathogenesis of OA.

Methods: Microarray datasets were obtained from the Gene Expression Omnibus (GEO) and ArrayExpress databases and were then analyzed using R software. To determine differential immune cell subtype infiltration, the CIBERSORT deconvolution algorithm was used. Quantitative reverse transcription PCR (qRT-PCR) was used to determine the relative expressions of selected genes. Besides, Western blotting was used to assess the protein expression levels in osteoarthritic chondrocytes.

Results: After analyzing the database profiles, two potential biomarkers, collagen type 3 alpha 1 chain (COL3A1), and matrix metalloproteinase 9 (MMP9), associated with OA were discovered, which were confirmed by qRT-PCR and Western blotting. Specifically, the results revealed that, as the concentration of $\mathrm{IL}-1 \beta$ increased, so did the gene and protein expression levels of COL3A1 and MMP9.

Conclusion: The findings provide valuable information and direction for future research into novel targets for OA immunotherapy and diagnosis and aids in the discovery of the underlying biological mechanisms of OA pathogenesis.

Keywords: osteoarthritis, immune cell infiltration, bioinformatics, GEO, diagnostic markers

\section{INTRODUCTION}

Osteoarthritis (OA), one of the most common age-related degenerative diseases, is characterized by osteophyte formation, cartilage degeneration, and synovial inflammation (Luo et al., 2018; Wang et al., 2018), which eventually lead to loss of joint function due to the limited repair capacity of the cartilage (Kim et al., 2018). However, the pathology of OA is not fully understood, and there 
is no treatment available to prevent or slow its progression (Wang et al., 2019). As a result, early diagnosis and treatment are preferred to improve joint function and alleviate joint pain.

According to recent research, the degenerative changes in the synovial membrane in OA occur earlier than those in the cartilage (Sakurai et al., 2019). OA synovitis is most likely caused by an innate immune response and is mediated by the expression of matrix-degrading enzymes, inflammatory cytokines, and chemokines (Gómez et al., 2015; Qadri et al., 2020). In several studies, the degree of synovitis has been validated as a strong predictor of OA, particularly in its early stages (Conaghan et al., 2010; Mathiessen and Conaghan, 2017). Immune responses are widely acknowledged to play an important role in the pathogenesis of OA (Daheshia and Yao, 2008; Han et al., 2018; Jenei-Lanzl et al., 2019). Pro-inflammatory cytokines promote chondrocyte apoptosis and cartilage matrix proteolysis (Utomo et al., 2016; Mobasheri et al., 2017). Furthermore, inflammatory suppression may aid in alleviating cartilage degradation in OA (Kapoor et al., 2011). However, the molecular mechanism of synovitis in the pathological process of OA has not been elucidated.

In the present study, microarray data from synovial membrane and cartilage samples in aged $\mathrm{OA}$ patients were integrated and the diagnostic biomarkers of OA were determined. The CIBERSORT algorithm method was then used to analyze immune cell infiltration in "normal" synovial membrane and OA synovial membrane. Furthermore, osteoarthritic chondrocytes $(\mathrm{OA}-\mathrm{CH})$ were stimulated with interleukin $1 \beta$ (IL-1 $\beta$ ) to establish a standardized in vitro OA model; the relationship between IL-1 $\beta$ and diagnostic biomarkers [collagen type 3 alpha 1 chain (COL3A1) and matrix metalloproteinase 9 (MMP9)] was determined by quantitative reverse transcription PCR (qRTPCR) and Western blotting. This study aimed to identify novel biomarkers associated with OA and to emphasize the importance of immune cells in the pathogenesis of OA. The findings of the current study could lead to new OA diagnostic targets.

\section{MATERIALS AND METHODS}

\section{Identification of Differentially Expressed Genes}

Figure 1 depicts the study workflow. Microarray datasets of synovial membrane (GSE55235 and GSE55457) and cartilage (GSE117999, GSE1919, GSE51588, and E-MTAB-5564) samples were obtained from the Gene Expression Omnibus (GEO) ${ }^{1}$ and ArrayExpress ${ }^{2}$ databases. The ComBat function in the sva

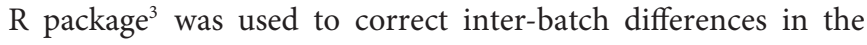
different datasets. The limma package $\mathrm{e}^{4}$ in $\mathrm{R}$ was used to normalize and screen differentially expressed genes (DEGs) by comparing the expression levels in the synovial membrane from normal

\footnotetext{
${ }^{1}$ http://www.ncbi.nlm.nih.gov/geo/

${ }^{2}$ https://www.ebi.ac.uk/arrayexpress/

${ }^{3}$ https://bioconductor.org/packages/release/bioc/html/sva.html

${ }^{4}$ http://bioconductor.org/packages/release/bioc/html/limma.html
}

joints to those from OA joints. DEGs with $|\log \mathrm{FC}|>2$ and an adjusted $p$-value $<0.05$ were considered significantly expressed.

\section{GO and KEGG Pathway Enrichment}

The cluster Profiler ${ }^{5}$ in $\mathrm{R}$ package was used to perform Gene Ontology (GO) annotation and Kyoto Encyclopedia of Genes and Genomes (KEGG) pathway enrichment analysis of the enriched DEGs. A value of $p<0.05$ was considered statistically significant.

\section{Construction of a PPI Network and Analysis of Hub Genes}

A protein-protein interaction (PPI) network was established using STRING, ${ }^{6}$ an online PPI establishment tool. The genes with a combined score of 0.4 were selected and used to establish the PPI network. Furthermore, the Cytohub plugin in Cytoscape version $3.8 .0^{7}$ was used to identify hub genes using the degree method (degree $>4$ ).

\section{CIBERSORT Analysis of Immune Cell Infiltration}

The CIBERSORT deconvolution algorithm ${ }^{8}$ was used to determine differential immune cell subtype infiltration between normal and OA synovial membrane samples. The difference in immune cell density between the normal and rheumatoid arthritis (RA) groups was visualized using a heatmap package in $\mathrm{R}$ version 3.6.0. The Wilcoxon signed-rank test was used to determine the statistical significance of the differences in immune cell infiltration between the two groups as depicted by violin plots.

\section{Ethical Statement}

The use of human material was approved by the local ethics committee of The First Affiliated Hospital of Zhengzhou University (reference no. 2021-KY-0338-002), and all patients provided written consent.

\section{IL-1 $\beta$ Stimulation of OA Chondrocytes}

Osteoarthritic chondrocytes $\left(2 \times 10^{5}\right.$, passages $\left.2-4\right)$ were cultured in six-well plates with DMEM F12 medium [supplemented with 10\% normal fetal calf serum (FCS) and $1 \%$ penicillin-streptomycin], stimulated with $\operatorname{IL}-1 \beta(1,5$, and $10 \mathrm{ng} / \mathrm{ml}$ ) (MAN0004230; Thermo Fisher Scientific, Waltham, MA, United States), and harvested for RNA and protein isolation after 24 and 48 h, respectively.

\section{RNA Extraction and Real-Time PCR Analysis}

Total RNA was isolated from the cells using the Absolutely RNA Miniprep Kit (Agilent Technologies, Santa Clara, CA, United States) according to the manufacturer's instructions and reverse-transcribed into complementary DNA (cDNA) using

\footnotetext{
${ }^{5} \mathrm{https} / / /$ www.bioconductor.org/help/search/index.html?q=clusterProfiler/

${ }^{6} \mathrm{http}: / /$ string-db.org

${ }^{7}$ https://cytoscape.org/

${ }^{8} \mathrm{https}: / /$ cibersort.stanford.edu/
} 


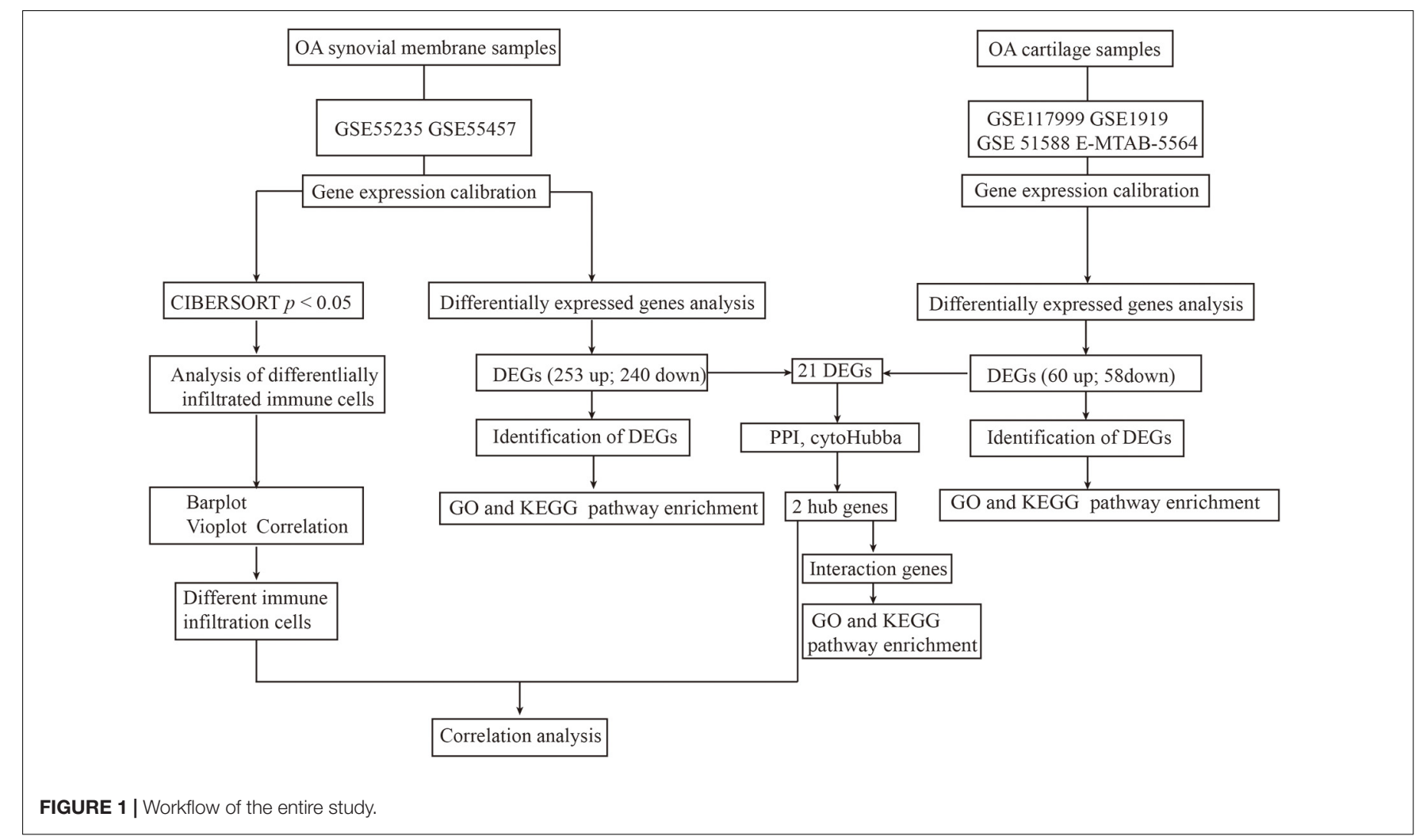

the AffinityScript QPCR cDNA Synthesis Kit (\#600559; Agilent Technologies, Santa Clara, CA, United States). Subsequently, an MX3005P QPCR System (Agilent Technologies, Santa Clara, CA, United States) was used to perform real-time PCR for messenger RNA (mRNA) expression with Brilliant III Ultra-Fast SYBR ${ }^{\circledR}$ Green QPCR Master Mix (\#600882; Agilent Technologies, Santa Clara, CA, United States). The primer sequences of the target genes were as follows: MMP9 (Fwd: 5'-GTA CCA CGG CCA ACT ACG AC-3'; Rev: $5^{\prime}$-GCC TTG GAA GAT GAA TGG AA-3'), COL3A1 (Fwd: 5'-CTTCTCTCCAGCCGAGCTTC3'; Rev: 5'-TGTGTTTCGTGCAACCATCC-3'), TBP (Fwd: 5'-TTGTAC CGCAGCTGCAAA AT-3'; Rev: 5'TATATTC GGCGTTTCGGGCA- ${ }^{\prime}$ ), and GAPDH (Fwd: 5'-CT GACTTCAACAGCGACACC-3'; Rev: 5'-CC CTGTTGCTGTAGCCAAAT-3'). All genes were analyzed relatively, calibrated to the expression of the control cell culture groups, and normalized to GAPDH and TBP.

\section{Protein Extraction and Western Blotting Analysis}

Osteoarthritic chondrocytes were washed twice with cold phosphate-buffered saline (PBS) and lysed with RIPA buffer (Thermo Fisher Scientific, Waltham, MA, United States) containing proteinase inhibitors (Roche, Basel, Switzerland). The concentration of cellular protein was determined using a BCA protein kit assay. Cell lysates were mixed with sodium dodecyl sulfate (SDS) sample loading buffer (\#B7053; Sigma-Aldrich, Taufkirchen, Germany), boiled for $5 \mathrm{~min}$ at $95^{\circ} \mathrm{C}$, and then subjected to $10 \%$ SDS-PAGE. After electrophoretic separation, the proteins were transferred to $0.22-\mathrm{mm}$ polyvinylidene fluoride (PVDF) membranes (Roche, Penzberg, Germany). Blot membranes were blocked with $5 \%$ bovine serum albumin (BSA) for $1 \mathrm{~h}$ at room temperature and incubated with primary antibodies on a shaker overnight at $4^{\circ} \mathrm{C}$. The membranes were then washed and incubated with the appropriate horseradish peroxidase-coupled secondary antibodies (Santa Cruz Biotechnology and Jackson ImmunoResearch, West Grove, PA, United States). The proteins were examined using enhanced chemiluminescence (ECL) detection reagents (Thermo Scientific, Waltham, MA, United States) and signals were normalized to $\beta$-actin. The following primary antibodies were used in this study: COL3A1 (1:1,000, \#ab838292; Abcam, Cambridge, MA, United States), MMP9 (1:200, \#sc-393859; Santa Cruz, Heidelberg, Germany), and $\beta$-actin (1:5,000, \#ab8227; Abcam, Cambridge, MA, United States).

\section{Statistical Analysis}

$\mathrm{R}$ version 3.6.0 was used to perform bioinformatics analyses, and a $p$-value $<0.05$ was considered statistically significant. Correlations were determined using Pearson's correlation coefficient, with $|R|<0.5$ indicating a weak correlation. For qRT-PCR and Western blotting analyses, an unpaired Student's $t$-test was used for two groups and one-way ANOVA was used for groups of more than two. Each assay was replicated and repeated in at least three independent experiments. A value of $p<0.05$ was considered statistically significant. 


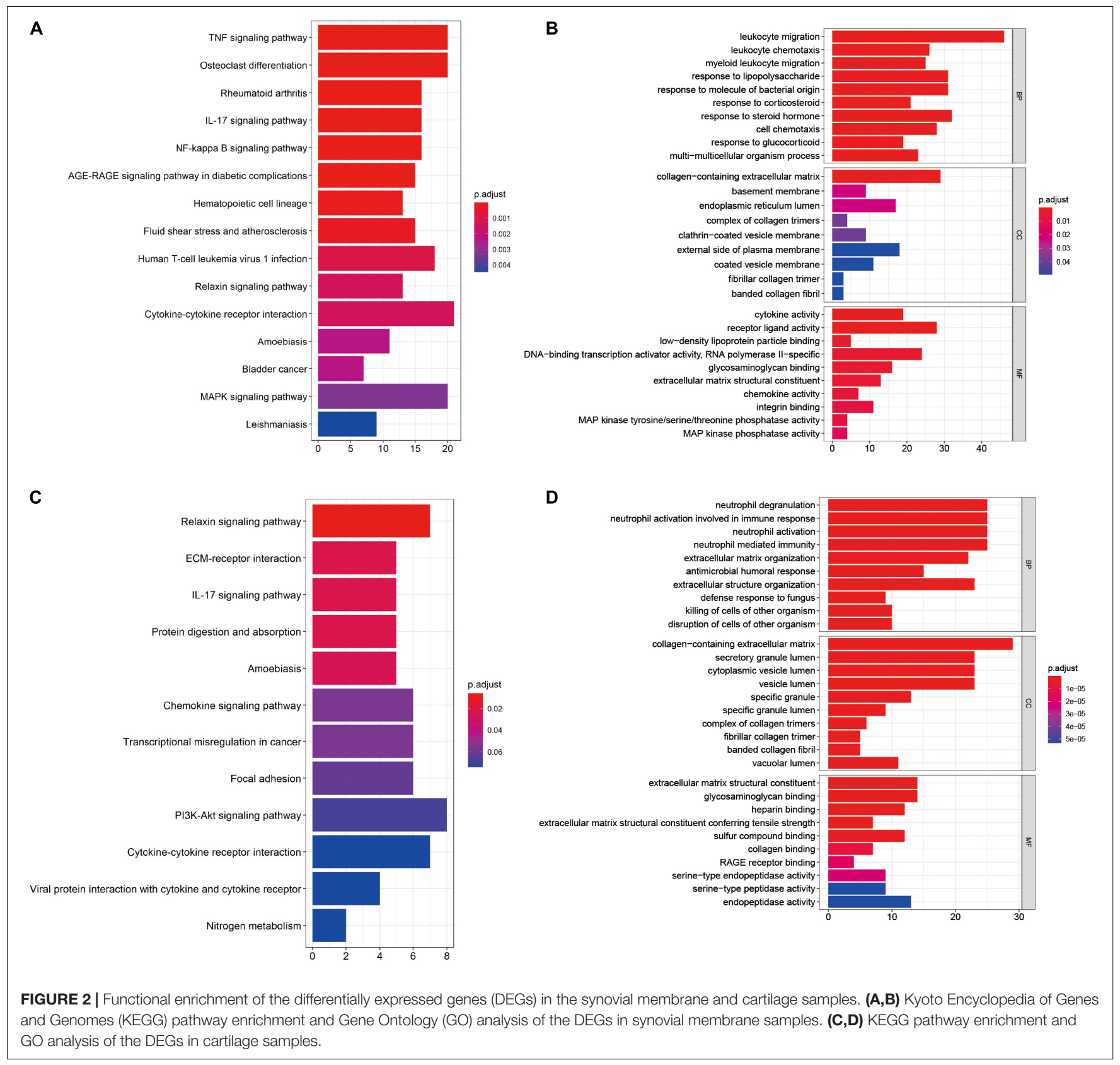

\section{RESULTS}

\section{Identification of DEGs}

Microarray datasets of synovial membrane (GSE55235 and GSE55457) and cartilage samples (GSE117999, GSE1919, GSE51588, and E-MTAB-5564) were obtained from the GEO and ArrayExpress databases. Before analyzing the DEGs, raw data were preprocessed for batch correction and normalization. Gene expression levels with $|\log \mathrm{FC}|>1$ and an adjusted $p$-value $<0.05$ were considered differentially expressed. As a result, 253 upregulated and 240 downregulated DEGs were identified in synovial membrane samples when compared to normal samples, while 60 upregulated and 58 downregulated
DEGs were identified in cartilage samples, as shown in Figure 1.

\section{Function Annotation of DEGs}

Kyoto Encyclopedia of Genes and Genomes pathway enrichment and GO functional enrichment of DEGs were performed to investigate the mechanisms involved in the pathogenesis of OA. KEGG pathway enrichment revealed that synovial membrane DEGs were mainly enriched in cytokine-cytokine receptor interaction, mitogen-activated protein kinase (MAPK) pathway, and tumor necrosis factor (TNF) pathway (Figure 2A), while DEGs from cartilage samples were enriched in the PI3K/AKT pathway, cytokine-cytokine receptor interaction, 

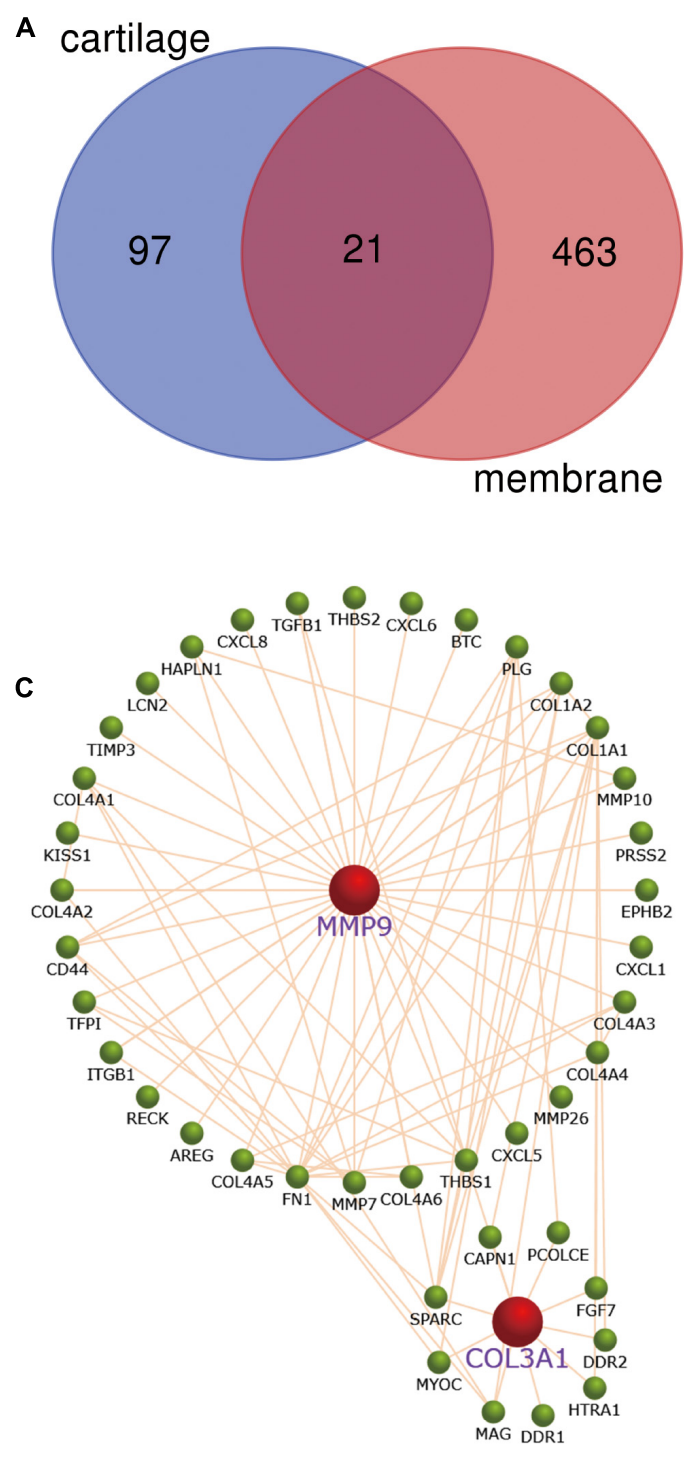

B
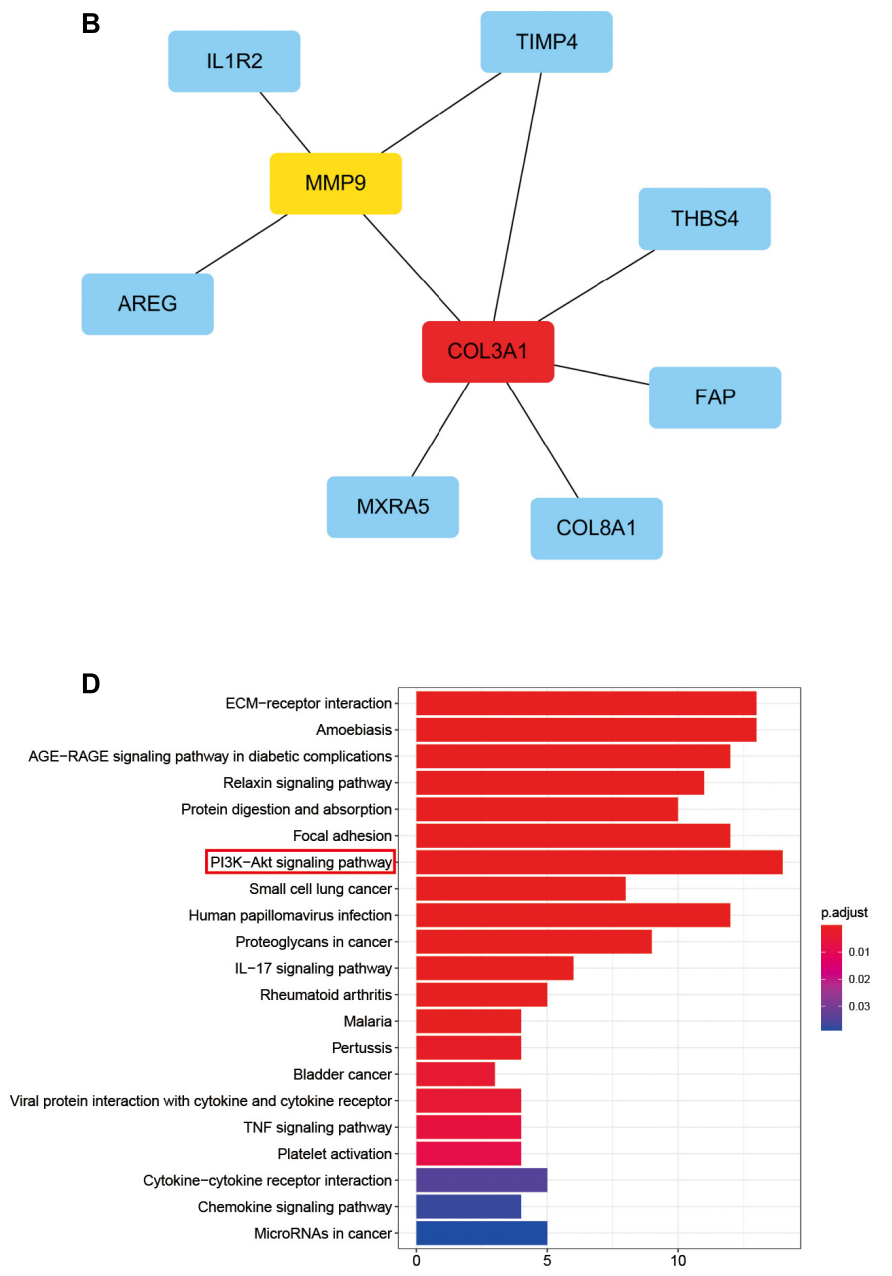

\footnotetext{
Hub genes

Interacting genes from dataset
}

FIGURE 3 | Screening of hub genes and functional analysis. (A) Twenty-one differentially expressed genes (DEGs) intersected between the cartilage samples and the synovial membrane samples. (B) Protein-protein interaction (PPI) network of the 21 DEGs and two hub genes screened by the degree method (degree $>4$ ) using cytoHubba. A higher ranking is represented by a redder color. (C) MMP9 and COL3A1 interacting genes indicated using Funrich software. (D) Kyoto Encyclopedia of Genes and Genomes (KEGG) pathway enrichment analysis of MMP9 and COL3A1 interacting genes.

and the chemokine pathway (Figure 2C). Furthermore, GO functional enrichment analysis revealed that synovial membrane DEGs were mainly involved in leukocyte migration, regulation of inflammatory response, and collagen-containing extracellular matrix (Figure 2B), while cartilage DEGs were mainly involved in the collagen-containing extracellular matrix, neutrophil degranulation, and neutrophil activation involved in immune response (Figure 2D). These findings suggest that DEGs in both the synovial membrane and cartilage are involved in immune response signaling pathways and that the immune system plays a critical role in the pathological processes of OA.

\section{Screening and Validation of MMP9 and COL3A1 Hub Genes}

The Venn diagram showed that 21 DEGs from the synovial membrane and cartilage samples overlapped (Figure $\mathbf{3 A}$ ). The 
TABLE 1 | Signaling pathway enrichment of MMP9 and COL3A1 interacting genes.

\begin{tabular}{|c|c|c|c|c|}
\hline ID & Description & $p$-value & $p_{\text {adjust }}$ & Count \\
\hline hsa04151 & PI3K-Akt signaling pathway & 4.70E-11 & $6.11 \mathrm{E}-10$ & 14 \\
\hline hsa04512 & ECM-receptor interaction & 9.39E-18 & 8.55E-16 & 13 \\
\hline hsa05146 & Amoebiasis & $7.07 \mathrm{E}-17$ & $3.22 \mathrm{E}-15$ & 13 \\
\hline hsa04933 & AGE-RAGE signaling pathway in diabetic complications & 2.89E-15 & 8.77E-14 & 12 \\
\hline hsa04510 & Focal adhesion & $1.38 \mathrm{E}-11$ & $2.09 E-10$ & 12 \\
\hline hsa05165 & Human papillomavirus infection & 4.48E-09 & 4.53E-08 & 12 \\
\hline hsa04926 & Relaxin signaling pathway & $2.35 \mathrm{E}-12$ & $5.35 E-11$ & 11 \\
\hline hsa04974 & Protein digestion and absorption & $7.43 \mathrm{E}-12$ & $1.35 E-10$ & 10 \\
\hline hsa05205 & Proteoglycans in cancer & $1.11 \mathrm{E}-07$ & $1.01 \mathrm{E}-06$ & 9 \\
\hline hsa05222 & Small cell lung cancer & 2.97E-09 & 3.38E-08 & 8 \\
\hline hsa04657 & IL-17 signaling pathway & 2.19E-06 & $1.81 \mathrm{E}-05$ & 6 \\
\hline hsa05323 & Rheumatoid arthritis & 3.88E-05 & 0.000295 & 5 \\
\hline hsa04060 & Cytokine-cytokine receptor interaction & 0.007225 & 0.034604 & 5 \\
\hline hsa05206 & MicroRNAs in cancer & 0.008991 & 0.03896 & 5 \\
\hline hsa05144 & Malaria & $5.26 \mathrm{E}-05$ & 0.000368 & 4 \\
\hline hsa05133 & Pertussis & 0.000271 & 0.001763 & 4 \\
\hline hsa04061 & Viral protein interaction with cytokine and cytokine receptor & 0.000772 & 0.004389 & 4 \\
\hline hsa04668 & TNF signaling pathway & 0.00118 & 0.006314 & 4 \\
\hline hsa04611 & Platelet activation & 0.001719 & 0.008692 & 4 \\
\hline hsa04062 & Chemokine signaling pathway & 0.008221 & 0.037406 & 4 \\
\hline hsa05219 & Bladder cancer & 0.000652 & 0.003953 & 3 \\
\hline
\end{tabular}

PPI network between the overlapping DEGs was constructed and two hub genes, MMP9 and COL3A1, were filtered out (Figure 3B) by the degree method (degree $>4$ ) using cytoHubba. The Funrich software was used to display the 41 interacting genes to better understand the functions of MMP9 and COL3A1 (Figure 3C). In addition, KEGG enrichment revealed that $41 M M P 9$ and COL3A1 interacting genes were involved in the PI3K/AKT pathway, IL-17 pathway, TNF pathway, and other immunerelated pathways (Figure 3D and Table 1). These findings imply that MMP9 and COL3A1 are involved in the pathophysiological inflammatory processes that lead to OA.

\section{Analysis of Immune Cell Infiltration in Normal and OA Synovial Membrane Samples}

The CIBERSORT algorithm was, for the first time, used to reveal the landscape of the differentially infiltrated immune cells in "normal" versus OA synovial membrane samples in 22 subpopulations of immune cells. The heatmap shows the proportion of immune cells in the two groups (Figure 4A).

The correlation heatmap of the 22 immune cell subtypes showed that two pairs of immune cells [active natural killer (NK) cells and eosinophils, and naive CD4 T cells and resting NK cells] were positively correlated and that two immune cell subtypes (activated mast cells and resting mast cells) were negatively correlated (Figure 4B).

Furthermore, the violin plot of the differentially infiltrated immune cells showed that regulatory $\mathrm{T}$ cells (Tregs) and resting mast cells had the highest infiltration rates in $\mathrm{OA}$ samples compared with "normal" samples, whereas resting $\mathrm{CD} 4{ }^{+}$ memory T cells, activated NK cells, activated mast cells, and eosinophils were less prominent in OA samples (Figures 5A, 6B).

\section{Correlation Between Hub Genes (MMP9 and COL3A1) and Immune Cell Infiltration}

Spearman's correlation analysis was performed to determine the association between the hub genes (MMP9 and COL3A1) and the infiltrated immune cell subtypes in the synovial membranes of both groups (Figure 5B). MMP9 and COL3A1 were found to be negatively correlated with resting CD4 memory T cells, whereas MMP9 was found to be positively correlated with M0 macrophages and negatively correlated with activated NK cells.

\section{Validation of Hub Genes (COL3A1 and MMP9) by qRT-PCR and Western Blotting}

The fragments per kilobase of exon model per million mapped fragments (FPKM) values of COL3A1 and MMP9 were significantly higher in the OA cartilage and synovial membrane compared with those in normal samples (Figures 6A,B). To validate the expressions of COL3A1 and MMP9 in chondrocytes, qRT-PCR and Western blotting were used to determine the gene and protein expressions in nonosteoarthritic chondrocytes $(\mathrm{NCH}), \mathrm{OA}-\mathrm{CH}$, and $\mathrm{OA}-\mathrm{CH}$ treated with different concentrations of IL-1 $\beta$. As shown in Figures 6C,D, the gene expression levels of both COL $3 A 1$ and MMP9 increased in the OA-CH group, and IL- $1 \beta$ promoted the expressions of COL $3 A 1$ and MMP9. Notably, the expressions of COL3A1 and MMP9 increased as the 


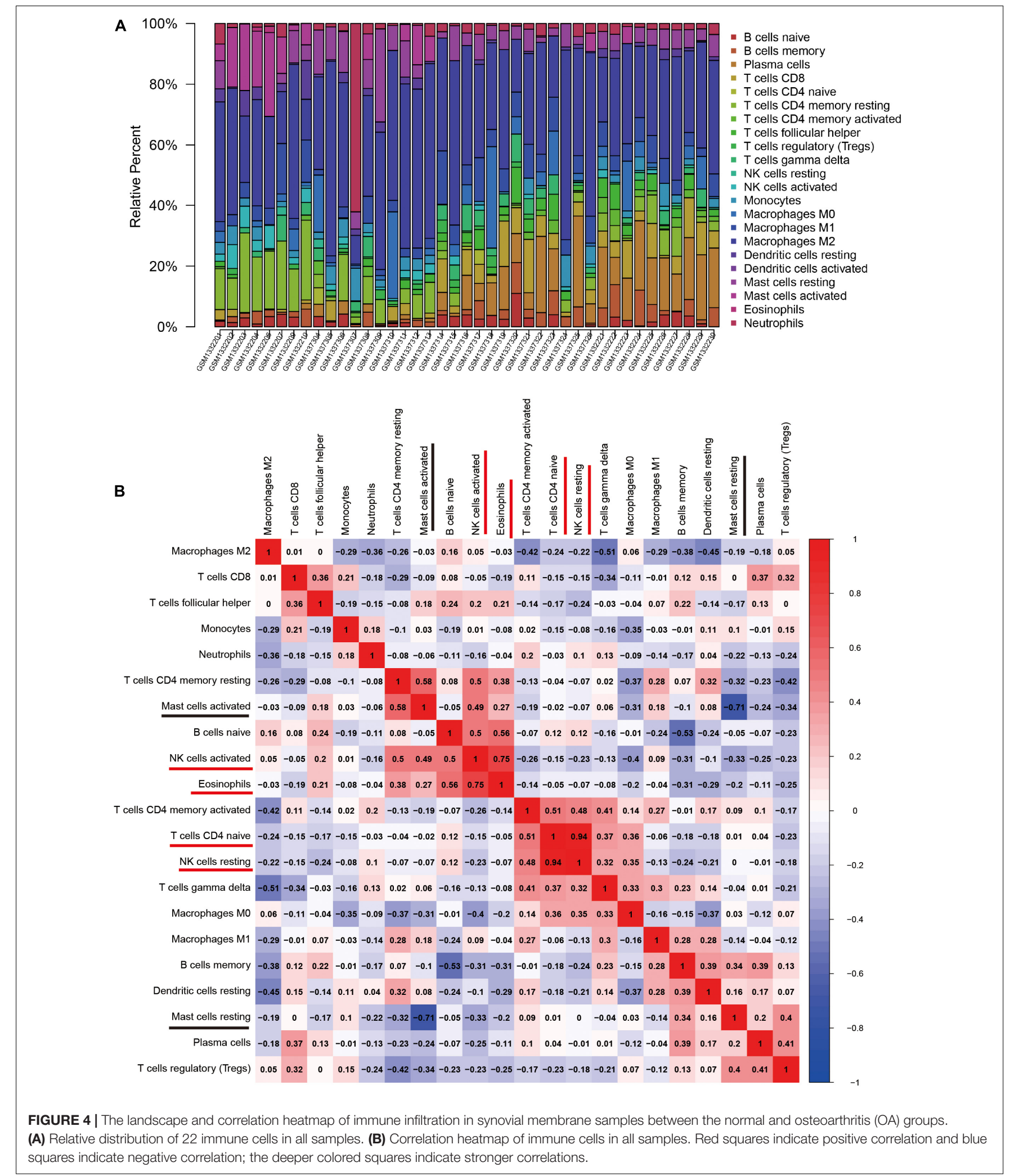

concentration of IL-1 $\beta$ increased. Furthermore, in the presence of IL-1 $\beta$, the protein levels of COL3A1 and MMP9 increased (Figures 6E-G). These findings suggest that the gene and protein expression levels of COL3A1 and MMP9 were positively correlated with the degree of inflammation and the inflammatory activity. 


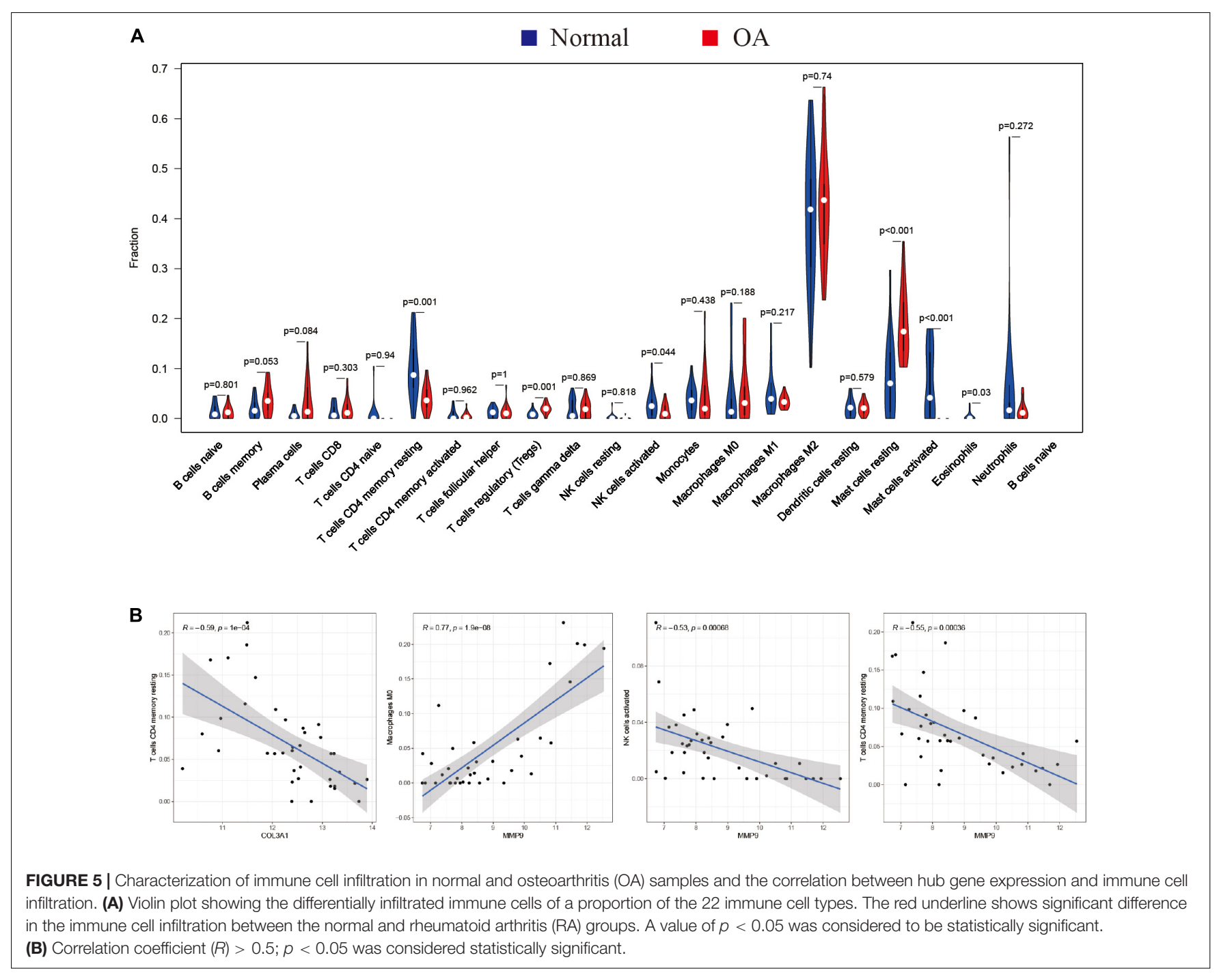

\section{DISCUSSION}

Osteoarthritic is a type of chronic joint disease that is characterized by cartilage degeneration, hyperosteogenia, and synovitis (Xie and Chen, 2019). Accumulating evidence suggests that pro-inflammatory cytokines, such as IL-1 $\beta$, TNF, and IL-6, play a role in the pathophysiology of OA (Robinson et al., 2016; Urban and Little, 2018). Previous research has focused on the molecular mechanism of $\mathrm{OA}$ in the cartilage or chondrocytes while ignoring the synovial membrane. In recent years, an increasing number of studies have shown that synovitis plays a critical role in the pathological process of OA, from the early to the end stages (Atukorala et al., 2016; Huang et al., 2018; Griffin and Scanzello, 2019). Additional research has revealed changes in immune cell infiltration in OA synovial membrane samples (Moradi et al., 2014; Penatti et al., 2017; Rosshirt et al., 2019). However, no study has been conducted to investigate the inflammatory relationship between the synovial membrane and cartilage. In the present study, the gene expression profiles of the synovial membrane and cartilage were combined to identify the important hub genes associated with synovitis in OA.

Differentially expressed genes in the synovial membrane and cartilage were separately analyzed; GO annotation and KEGG pathway enrichment were used to reveal the functions of these DEGs. Our results also showed that both synovial membrane and cartilage DEGs were mainly involved in inflammatory pathways and pathological processes, which was consistent with previous studies (Qin et al., 2012; Hou et al., 2013; Chen et al., 2018). Furthermore, the immune response occurred in the synovial membrane and cartilage, indicating that synovitis plays a critical role in the pathological process of OA.

The hub genes COL3A1 and MMP9 were identified and their function validated using Funrich software and by KEGG pathway enrichment, respectively. Besides, COL3A1 and MMP9 interacting genes were found to be mainly involved in the PI3K/AKT signaling pathway, extracellular matrix (ECM) receptor interaction, and other inflammatory signaling pathways (IL-17 signaling pathway, cytokine-cytokine receptor interaction, TNF signaling pathway, and chemokine signaling 
A

A $\quad$ MMP9

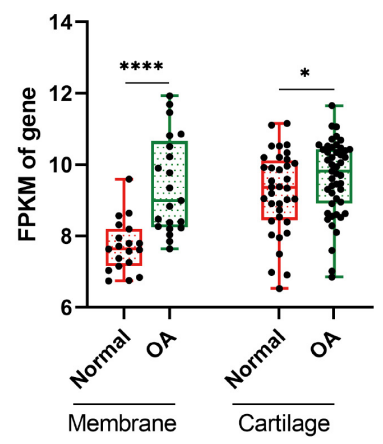

B

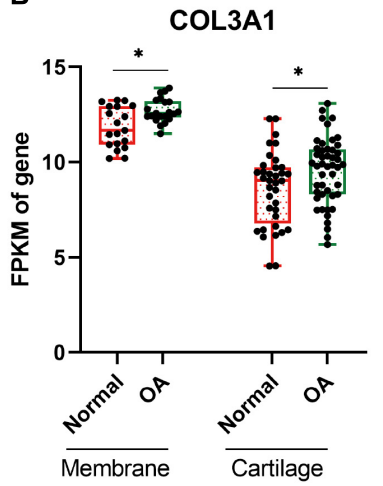

C
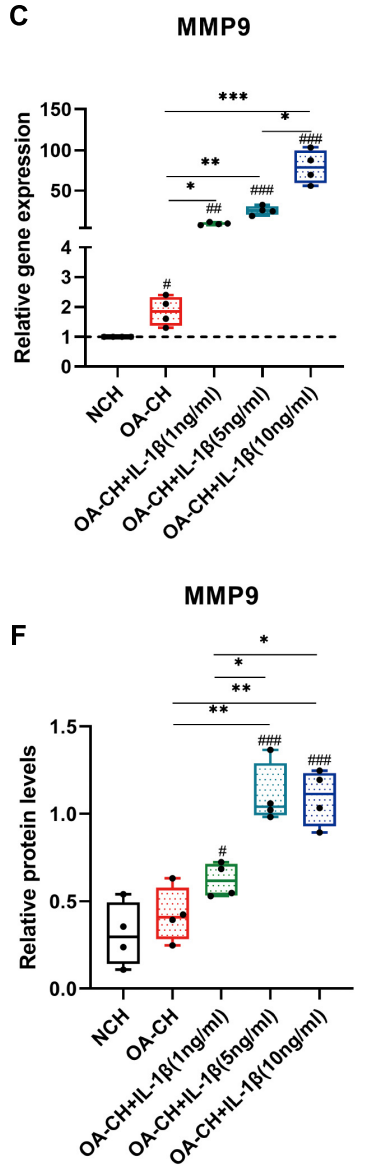

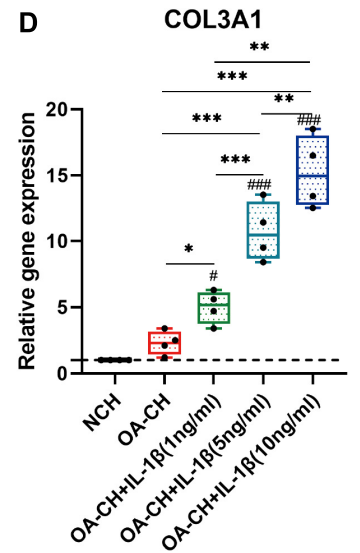

COL3A1

G

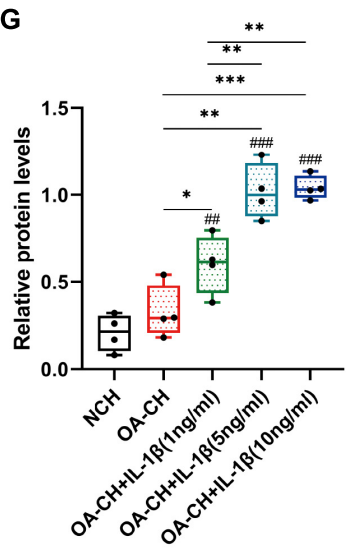

FIGURE 6 | Validation of the hub genes (COL3A1 and MMP9) by quantitative reverse transcription PCR (qRT-PCR) and Western blotting. (A,B) Fragments per kilobase of exon model per million mapped fragments (FPKM) of COL3A1 and MMP9 in synovial membrane and cartilage samples. ${ }^{*} p<0.05$; ${ }^{* * * *} p<0.001$, unpaired Student's $t$-test. (C,D) Gene expression levels of COL3A1 and MMP9 in non-osteoarthritic chondrocytes (NCH) and osteoarthritic chondrocytes (OA-CH) treated with different concentrations of IL-1 1 . (E-G) Western blotting was used to determine the protein expression levels of COL3A1 and MMP9 in NCH and OA-CH treated with different concentrations of IL-1 $\beta$. Significant difference to control $(\mathrm{NCH})$ : $\# p<0.05$; \#\# $p<0.01$; \#\#\# $p<0.001$. *Significant difference between groups: ${ }^{*} p<0.05 ;{ }^{* *} p<0.01 ;{ }^{* * *} p<0.001$. One-way ANOVA with Newman-Keuls multiple comparison test. All values represent the mean \pm standard deviation $(n=4)$.

pathway). Numerous studies have reported that inflammatory signaling pathways, including the PI3K/AKT, IL-17, TNF, NF-KB, and MAPK signaling pathways, are involved in the osteoarthritic process (Balabko et al., 2015; Zhang et al., 2018; Han et al., 2019; Li and Zheng, 2019). These findings suggest that COL3A1 and $M M P 9$ play important roles in the inflammatory signaling pathways linked to OA.

To further investigate the effect of immune cell infiltration in OA, CIBERSORT was used to perform a comprehensive analysis of OA immune infiltration. The results showed increased infiltration of Tregs and resting mast cells, which contributed to the occurrence and development of OA. Moradi et al. (2014) found that Tregs are enriched in the synovial membrane of OA patients and correlated with the levels of inflammatory factors (IL-10 and TGF- $\beta$ ) (Xia et al., 2017). Resting mast cells were found in high numbers in OA synovial tissue, which is associated with structural damage in OA patients (de Lange-Brokaar et al., 2016). These findings and other related research indicate that Tregs and resting mast cells play an important role in OA. In this study, the relationship between the immune cell subtypes in OA was investigated; the results showed that two pairs of immune cells (activated NK cells and eosinophils, and naive $\mathrm{CD} 4 \mathrm{~T}$ cells and resting NK cells) were positively correlated and that two immune cell subtypes (activated and resting mast cells) were negatively correlated. However, the correlation between the immune cell subtypes requires further experimental validation.

The relationship between hub gene expression and immune cell infiltration was also analyzed. The results showed that the expressions of both $M M P 9$ and COL3A1 were negatively correlated with resting CD4 memory T cells, while the expression of MMP9 was positively correlated with M0 macrophages and negatively correlated with activated NK cells. We hypothesized that MMP9 and COL3A1 inhibited the immune response by reducing the resting $\mathrm{CD} 4$ memory $\mathrm{T}$ cells and activated $\mathrm{NK}$ cells and that MMP9 increased M0 macrophages to induce inflammation in the course of OA. However, further research is needed to validate these assumptions on the relationship between hub genes and immune cells. 
To investigate the correlation between the hub genes (MMP9 and COL3A1) and OA, qRT-PCR, and Western blotting were used to determine the gene and protein expression levels in chondrocytes. The results indicated that the gene expression levels of MMP9 and COL3A1 increased in $\mathrm{OA}-\mathrm{CH}$ compared with those in $\mathrm{NCH}$. Notably, the gene and protein expression levels of COL $3 A 1$ and MMP9 increased with an increase in IL-1 $\beta$ concentration. Evidence suggests that the expression levels of COL3A1 increased in the early stages of OA and decreased in the later stages (Rai et al., 2019). Tang et al. (2018) also discovered that IL-1 increased the protein levels of COL $3 A 1$ in synoviocytes. MMP9, also known as gelatinase $B$, is an enzyme that degrades the ECM components such as collagen, fibronectin, and laminin. MMP9 was found to be upregulated at the mRNA and protein levels in the cartilage and synovial membrane, as well as in the synovial fluid, and was found to be related to the severity of OA (Bollmann et al., 2021). These findings suggest that COL3A1 and MMP9 could be used as OA diagnostic biomarkers. However, more research is needed to determine the roles of COL3A1 and MMP9 in the progression of OA.

\section{CONCLUSION}

In conclusion, the present study identified two potential OA biomarkers, COL3A1, and MMP9, which were confirmed by qRTPCR and Western blotting analysis. Notably, the gene and protein expression levels of COL3A1 and MMP9 increased with an increase in IL- $1 \beta$ concentration. These findings provide valuable information and direction for future research into novel targets for OA immunotherapy and diagnosis and aid in the discovery of the underlying biological mechanisms of OA pathogenesis.

\section{REFERENCES}

Atukorala, I., Kwoh, C. K., Guermazi, A., Roemer, F. W., Boudreau, R. M., Hannon, M. J., et al. (2016). Synovitis in knee osteoarthritis: a precursor of disease? Ann. Rheum. Dis. 75, 390-395. doi: 10.1136/annrheumdis-2014-205894

Balabko, L., Andreev, K., Burmann, N., Schubert, M., Mathews, M., Trufa, D. I., et al. (2015). Increased expression of the Th17-IL-6R/pSTAT3/BATF/Ror $\gamma$ Taxis in the tumoural region of adenocarcinoma as compared to squamous cell carcinoma of the lung. Sci. Rep. 4:7396. doi: 10.1038/srep07396

Bollmann, M., Pinno, K., Ehnold, L. I., Märtens, N., Märtson, A., Pap, T., et al. (2021). MMP-9 mediated Syndecan-4 shedding correlates with osteoarthritis severity. Osteoarthritis Cartilage 29, 280-289. doi: 10.1016/j.joca.2020.10.009

Chen, Y., Shou, K., Gong, C., Yang, H., Yang, Y., and Bao, T. (2018). Anti-inflammatory effect of geniposide on osteoarthritis by suppressing the activation of p38 MAPK signaling pathway. Biomed. Res. Int. 2018:8384576. doi: $10.1155 / 2018 / 8384576$

Conaghan, P. G., D’Agostino, M. A., Le Bars, M., Baron, G., Schmidely, N., Wakefield, R., et al. (2010). Clinical and ultrasonographic predictors of joint replacement for knee osteoarthritis: results from a large, 3-year, prospective EULAR study. Ann. Rheum. Dis. 69, 644-647. doi: 10.1136/ard.2008.099564

Daheshia, M., and Yao, J. Q. (2008). The interleukin lbeta pathway in the pathogenesis of osteoarthritis. J. Rheumatol. 35, 2306-2312. doi: 10.3899/ jrheum.080346

de Lange-Brokaar, B. J. E., Kloppenburg, M., Andersen, S. N., Dorjée, A. L., Yusuf, E., Herb-van Toorn, L., et al. (2016). Characterization of synovial mast cells in knee osteoarthritis: association with clinical parameters. Osteoarthritis Cartilage 24, 664-671. doi: 10.1016/j.joca.2015.11.011

\section{DATA AVAILABILITY STATEMENT}

The original contributions presented in the study are included in the article/supplementary material, further inquiries can be directed to the corresponding author.

\section{ETHICS STATEMENT}

The use of human material has been approved by the local ethics committee (reference number: 2021-KY-0338-002, The First Affiliated Hospital of Zhengzhou University) and the written consent of all patients has been obtained.

\section{AUTHOR CONTRIBUTIONS}

SL contributed to sample collection, experiment design, investigation, data curation, writing the original draft, and review and editing. $\mathrm{HW}$ and $\mathrm{YZ}$ helped with conceptualization and review and editing. RQ, PX, HZ, and ZK helped with the methodology, establishment of qRT-PCR, and bioinformatics analysis. LY made contributions to the conceptualization, review and editing, project administration, and funding acquisition. All authors proofread the final version of the manuscript.

\section{FUNDING}

This study was funded by the Key Scientific Research Projects of Colleges and Universities in Henan Province (20A310022).

Gómez, R., Villalvilla, A., Largo, R., Gualillo, O., and Herrero-Beaumont, G. (2015). TLR4 signalling in osteoarthritis-finding targets for candidate DMOADs. Nat. Rev. Rheumatol. 11, 159-170. doi: 10.1038/nrrheum.2014.209

Griffin, T. M., and Scanzello, C. R. (2019). Innate inflammation and synovial macrophages in osteoarthritis pathophysiology. Clin. Exp. Rheumatol. 37(Suppl. 120), 57-63.

Han, P.-F., Wei, L., Duan, Z.-Q., Zhang, Z.-L., Chen, T.-Y., Lu, J.-G., et al. (2018). Contribution of IL-1 $\beta, 6$ and TNF- $\alpha$ to the form of post-traumatic osteoarthritis induced by "idealized" anterior cruciate ligament reconstruction in a porcine model. Int. Immunopharmacol. 65, 212-220. doi: 10.1016/j.intimp.2018. 10.007

Han, Y., Li, X., Yan, M., Yang, M., Wang, S., Pan, J., et al. (2019). Oxidative damage induces apoptosis and promotes calcification in disc cartilage endplate cell through ROS/MAPK/NF-кB pathway: implications for disc degeneration. Biochem. Biophys. Res. Commun. 516, 1026-1032. doi: 10.1016/j.bbrc.2017.03. 111

Hou, C.-H., Tang, C.-H., Hsu, C.-J., Hou, S.-M., and Liu, J.-F. (2013). CCN4 induces IL-6 production through $\alpha \mathrm{v} \beta 5$ receptor, PI3K, Akt, and NF- $\kappa \mathrm{B}$ singling pathway in human synovial fibroblasts. Arthritis Res. Ther. 15:R19. doi: 10.1186/ ar4151

Huang, H., Zheng, J., Shen, N., Wang, G., Zhou, G., Fang, Y., et al. (2018). Identification of pathways and genes associated with synovitis in osteoarthritis using bioinformatics analyses. Sci. Rep. 8:10050. doi: 10.1038/s41598-01828280-6

Jenei-Lanzl, Z., Meurer, A., and Zaucke, F. (2019). Interleukin-1 $\beta$ signaling in osteoarthritis-chondrocytes in focus. Cell. Signal. 53, 212-223. doi: 10.1016/j. cellsig.2018.10.005 
Kapoor, M., Martel-Pelletier, J., Lajeunesse, D., Pelletier, J.-P., and Fahmi, H. (2011). Role of proinflammatory cytokines in the pathophysiology of osteoarthritis. Nat. Rev. Rheumatol. 7, 33-42. doi: 10.1038/nrrheum.2010.196

Kim, J.-R., Yoo, J. J., and Kim, H. A. (2018). Therapeutics in osteoarthritis based on an understanding of its molecular pathogenesis. Int. J. Mol. Sci. 19:674. doi: 10.3390/ijms19030674

Li, J., and Zheng, J. (2019). Theaflavins prevent cartilage degeneration via AKT/FOXO3 signaling in vitro. Mol. Med. Rep. 19, 821-830. doi: 10.3892/mmr. 2018.9745

Luo, Y., He, Y., Reker, D., Gudmann, N. S., Henriksen, K., Simonsen, O., et al. (2018). A novel high sensitivity type II collagen blood-based biomarker, PROC2, for assessment of cartilage formation. Int. J. Mol. Sci. 19:3485. doi: 10.3390/ ijms 19113485

Mathiessen, A., and Conaghan, P. G. (2017). Synovitis in osteoarthritis: current understanding with therapeutic implications. Arthritis Res. Ther. 19:18. doi: 10.1186/s13075-017-1229-9

Mobasheri, A., Rayman, M. P., Gualillo, O., Sellam, J., van der Kraan, P., and Fearon, U. (2017). The role of metabolism in the pathogenesis of osteoarthritis. Nat. Rev. Rheumatol. 13, 302-311. doi: 10.1038/nrrheum.2017.50

Moradi, B., Schnatzer, P., Hagmann, S., Rosshirt, N., Gotterbarm, T., Kretzer, J. P., et al. (2014). CD4+CD25+/highCD127low/- regulatory $\mathrm{T}$ cells are enriched in rheumatoid arthritis and osteoarthritis joints-analysis of frequency and phenotype in synovial membrane, synovial fluid and peripheral blood. Arthritis Res. Ther. 16:R97. doi: 10.1186/ar4545

Penatti, A., Facciotti, F., De Matteis, R., Larghi, P., Paroni, M., Murgo, A., et al. (2017). Differences in serum and synovial CD4+ T cells and cytokine profiles to stratify patients with inflammatory osteoarthritis and rheumatoid arthritis. Arthritis Res. Ther. 19:103. doi: 10.1186/s13075-017-1305-1

Qadri, M., Jay, G. D., Zhang, L. X., Richendrfer, H., Schmidt, T. A., and Elsaid, K. A. (2020). Proteoglycan-4 regulates fibroblast to myofibroblast transition and expression of fibrotic genes in the synovium. Arthritis Res. Ther. 22:113. doi: 10.1186/s13075-020-02207-x

Qin, J., Shang, L., Ping, A., Li, J., Li, X., Yu, H., et al. (2012). TNF/TNFR signal transduction pathway-mediated anti-apoptosis and anti-inflammatory effects of sodium ferulate on IL-1 $\beta$-induced rat osteoarthritis chondrocytes in vitro. Arthritis Res. Ther. 14:R242. doi: 10.1186/ar4085

Rai, M. F., Tycksen, E. D., Cai, L., Yu, J., Wright, R. W., and Brophy, R. H. (2019). Distinct degenerative phenotype of articular cartilage from knees with meniscus tear compared to knees with osteoarthritis. Osteoarthritis Cartilage 27, 945-955. doi: 10.1016/j.joca.2019.02.792

Robinson, W. H., Lepus, C. M., Wang, Q., Raghu, H., Mao, R., Lindstrom, T. M., et al. (2016). Low-grade inflammation as a key mediator of the pathogenesis of osteoarthritis. Nat. Rev. Rheumatol. 12, 580-592. doi: 10.1038/nrrheum.2016. 136

Rosshirt, N., Hagmann, S., Tripel, E., Gotterbarm, T., Kirsch, J., Zeifang, F., et al. (2019). A predominant Th1 polarization is present in synovial fluid of endstage osteoarthritic knee joints: analysis of peripheral blood, synovial fluid and synovial membrane. Clin. Exp. Immunol. 195, 395-406. doi: 10.1111/cei.13230

Sakurai, Y., Fujita, M., Kawasaki, S., Sanaki, T., Yoshioka, T., Higashino, K., et al. (2019). Contribution of synovial macrophages to rat advanced osteoarthritis pain resistant to cyclooxygenase inhibitors. Pain 160, 895-907. doi: 10.1097/j. pain.0000000000001466

Tang, S., Deng, S., Guo, J., Chen, X., Zhang, W., Cui, Y., et al. (2018). Deep coverage tissue and cellular proteomics revealed IL-1 $\beta$ can independently induce the secretion of TNF-associated proteins from human synoviocytes. J. Immunol. 200, 821-833. doi: 10.4049/jimmunol.1700480

Urban, H., and Little, C. B. (2018). The role of fat and inflammation in the pathogenesis and management of osteoarthritis. Rheumatology (Oxford) 57, iv10-iv21. doi: 10.1093/rheumatology/kex399

Utomo, L., Bastiaansen-Jenniskens, Y. M., Verhaar, J. A. N., and van Osch, G. J. V. M. (2016). Cartilage inflammation and degeneration is enhanced by pro-inflammatory (M1) macrophages in vitro, but not inhibited directly by anti-inflammatory (M2) macrophages. Osteoarthritis Cartilage 24, 2162-2170. doi: 10.1016/j.joca.2016.07.018

Wang, Q., Onuma, K., Liu, C., Wong, H., Bloom, M. S., Elliott, E. E., et al. (2019). Dysregulated integrin $\alpha \mathrm{V} \beta 3$ and CD47 signaling promotes joint inflammation, cartilage breakdown, and progression of osteoarthritis. JCI Insight 4:e128616. doi: $10.1172 /$ jci.insight. 128616

Wang, R., Xu, B., and Xu, H. (2018). TGF- $\beta 1$ promoted chondrocyte proliferation by regulating Sp1 through MSC-exosomes derived miR-135b. Cell Cycle 17, 2756-2765. doi: 10.1080/15384101.2018.1556063

Xia, J., Ni, Z., Wang, J., Zhu, S., and Ye, H. (2017). Overexpression of lymphocyte activation gene-3 inhibits regulatory $\mathrm{T}$ Cell responses in osteoarthritis. DNA Cell Biol. 36, 862-869. doi: 10.1089/dna.2017.3771

Xie, C., and Chen, Q. (2019). Adipokines: new therapeutic target for osteoarthritis? Curr. Rheumatol. Rep. 21:71. doi: 10.1007/s11926-0190868-Z

Zhang, W., Hsu, P., Zhong, B., Guo, S., Zhang, C., Wang, Y., et al. (2018). MiR-34a enhances chondrocyte apoptosis, senescence and facilitates development of osteoarthritis by targeting DLL1 and regulating PI3K/AKT pathway. Cell. Physiol. Biochem. 48, 1304-1316. doi: 10.1159/00049 2090

Conflict of Interest: The authors declare that the research was conducted in the absence of any commercial or financial relationships that could be construed as a potential conflict of interest.

Publisher's Note: All claims expressed in this article are solely those of the authors and do not necessarily represent those of their affiliated organizations, or those of the publisher, the editors and the reviewers. Any product that may be evaluated in this article, or claim that may be made by its manufacturer, is not guaranteed or endorsed by the publisher.

Copyright (c) 2021 Li, Wang, Zhang, Qiao, Xia, Kong, Zhao and Yin. This is an open-access article distributed under the terms of the Creative Commons Attribution License (CC BY). The use, distribution or reproduction in other forums is permitted, provided the original author(s) and the copyright owner(s) are credited and that the original publication in this journal is cited, in accordance with accepted academic practice. No use, distribution or reproduction is permitted which does not comply with these terms. 